\title{
Detection of Influenza Virus Infection Using Two PCR Methods
}

\author{
Richard K. Zimmerman, ${ }^{1}$ Charles R. Rinaldo, ${ }^{2,3}$ \\ Mary Patricia Nowalk, ${ }^{1}$ G. K. Balasubramani, ${ }^{4}$ Mark G. Thompson, ${ }^{5}$ \\ Arlene Bullotta, ${ }^{2}$ Michael Susick, ${ }^{1}$ and Stephen Wisniewski ${ }^{4}$ \\ ${ }^{1}$ Department of Family Medicine, University of Pittsburgh School of Medicine, 3518 5th Avenue, Pittsburgh, PA 15261, USA \\ ${ }^{2}$ Department of Pathology, University of Pittsburgh School of Medicine, PA 15261, USA \\ ${ }^{3}$ Department of Infectious Disease and Microbiology, University of Pittsburgh Graduate School of Public Health, Pittsburgh, \\ PA 15261, USA \\ ${ }^{4}$ Department of Epidemiology, University of Pittsburgh Graduate School of Public Health, Pittsburgh, PA 15261, USA \\ ${ }^{5}$ Influenza Division, Centers for Disease Control and Prevention, Atlanta, GA 30307, USA
}

Correspondence should be addressed to Mary Patricia Nowalk; tnowalk@pitt.edu

Received 15 September 2014; Accepted 21 November 2014; Published 9 December 2014

Academic Editor: Stefan Pöhlmann

Copyright (c) 2014 Richard K. Zimmerman et al. This is an open access article distributed under the Creative Commons Attribution License, which permits unrestricted use, distribution, and reproduction in any medium, provided the original work is properly cited.

Rapid, accurate, and cost-effective methods to identify the cause of respiratory tract infections are needed to maximize clinical benefit. Outpatients with acute respiratory illness were tested for influenza using a singleplex reverse transcriptase polymerase chain reaction (SRT-PCR) method. A multiplex RT-PCR (MRT-PCR) method tested for influenza and 17 other viruses and was compared with SRT-PCR using chi-square tests. Among 935 patients, 335 (36\%) tested positive for influenza A and influenza B using SRT-PCR. Using MRT-PCR, 320 (34.2\%) tested positive for influenza A and influenza B. This study supports MRT-PCR as a comparable method for detecting influenza among patients seeking outpatient care for acute respiratory illnesses.

\section{Background}

Each year millions of people are afflicted with influenzaassociated respiratory tract infections, some of which lead patients to seek outpatient medical attention, referred to as medically attended acute respiratory infection (ARI). Rapid, accurate, and cost-effective methods are needed to maximize clinical benefit of antivirals and reduce inappropriate antibiotic usage. New assay methods using multiplex reverse transcriptase polymerase chain reaction (MRT-PCR) are available that allow for relatively rapid detection of multiple virus types including influenza $[1,2]$.

\section{Objective}

During the 2012-2013 influenza season, the Centers for Disease Control and Prevention (CDC)-funded, multicenter US Influenza Vaccine Effectiveness (Flu VE) Network conducted a study designed to determine the effectiveness of the season's influenza vaccine. That study used singleplex RT-PCR
(SRT-PCR) to detect influenza virus. The University of Pittsburgh site of the Flu VE Network also used MRT-PCR. The purpose of this study was to compare the agreement between SRT-PCR and MRT-PCR for influenza virus detection.

\section{Study Design}

The US Flu VE Network evaluated the effectiveness of the season's influenza vaccine using a test-negative, case-control study design $[3,4]$, where the proportion vaccinated among those who test positive for influenza was compared with the proportion vaccinated among those who test negative. Participants in the current study were enrollees in the University of Pittsburgh site of the Flu VE study, described previously [5]. Eligibility criteria included patients aged $\geq 6$ months as of $9 / 1 / 2012$, seeking outpatient medical care for an upper respiratory illness of $\leq 7$ days' duration with cough, and not taking an influenza antiviral before the visit. This prospective study was approved by the University of Pittsburgh Institutional Review Board. 
TABLE 1: Comparison of singleplex reverse transcriptase polymerase chain reaction (SRT-PCT) with multiplex reverse transcriptase polymerase chain reaction (MRT-PCR) showing sensitivity and specificity of MRT-PCR compared to SRT-PCR for influenza, $2012-2013$.

\begin{tabular}{|c|c|c|c|c|c|c|c|}
\hline \multicolumn{4}{|c|}{ Influenza A } & \multicolumn{4}{|c|}{ Influenza B } \\
\hline \multirow{2}{*}{ MRT-PCR } & \multicolumn{3}{|c|}{ SRT-PCR } & \multirow{2}{*}{ MRT-PCR } & \multicolumn{3}{|c|}{ SRT-PCR } \\
\hline & Positive & Negative & Total & & Positive & Negative & Total \\
\hline Positive & 250 & 1 & 251 & Positive & 69 & 0 & 69 \\
\hline Negative & 10 & 672 & 682 & Negative & 7 & 851 & 858 \\
\hline Total & 260 & 673 & $933^{*}$ & Total & 76 & 851 & $927^{*}$ \\
\hline Sensitivity/specificity & $96 \%$ & $99.8 \%$ & & Sensitivity/specificity & $91 \%$ & $100 \%$ & \\
\hline
\end{tabular}

MRT-PCR: multiplex reverse transcriptase polymerase chain reaction; SRT-PCR: singleplex reverse transcriptase polymerase chain reaction.

${ }^{*}$ Of the 935 specimens, 2 specimens in SRT-PCR had inconclusive results/missing data (leaving 933) and were MRT-PCR negative for influenza A and 8 specimens in SRT-PCR had inconclusive results/missing data (leaving 927) and were MRT-PCR negative for influenza B.

Nasal and oropharyngeal mucosa were each sampled using polyester swabs that were combined in the same cryovial and delivered to the UPMC Clinical Virology Laboratory within 72 hours. The specimens were stored in a lysis buffer and aliquoted for nucleic acid isolation and detection of influenza virus using CDC's singleplex RT-PCR (SRTPCR) test and a MRT-PCR test using the eSensor XT-8 instrument and respiratory viral panel from GenMark Diagnostics, Inc. In toto, 1171 specimens were collected and tested for presence of influenza using SRT-PCR. Of those, funding was available to analyze with MRT-PCR all specimens SRT-PCRpositive for influenza and a random sample of specimens SRT-PCR-negative for influenza, for a total of 935 specimens that were doubly assayed. The purpose of this study was to compare the agreement between SRT-PCR and MRT-PCR for influenza virus detection.

Both the SRT-PCR and MRT-PCR extraction and assay methods have been previously published $[1,6,7]$. The eSensor RVP MRT-PCR assay (GenMark Diagnostics) used in this study is currently approved for clinical use in Europe. It has the same methodological characteristics but a broader range of viral analytes than the US FDA-cleared version and includes seasonal influenza A virus (H1N1 and H3N2 subtypes) and influenza B virus.

The measurement of agreement between the SRT-PCR and MRT-PCR influenza assays was determined using Cohen's kappa statistic. Analyses were conducted using SAS version 9.2 (SAS Institute, Inc., Cary, NC).

\section{Results}

Among 935 ARI patients, influenza A virus was detected in 260 specimens and influenza B virus was detected in 76 specimens using SRT-PCR, and influenza A virus was detected in 251 specimens and influenza B virus was detected in 69 specimens using MRT-PCR. There was significant agreement on influenza results between assays; for influenza A, kappa $=0.97,95 \%$ confidence intervals $(95 \% \mathrm{CI})=0.95-$ 0.99 , and Chi-square $P<0.001$; for influenza $\mathrm{B}$, kappa $=$ $0.95,95 \% \mathrm{CI}=0.91-0.99$, and Chi-square $P<0.001$; and for overall results, kappa $=0.96,95 \% \mathrm{CI}=0.94-0.98$, and Chi-square $P<0.001$. The sensitivity was $96 \%$ and $91 \%$ and specificity was $99.8 \%$ and $100 \%$, respectively, for influenza
A and influenza B, when SRT-PCR was treated as the gold standard (Table 1).

The mean cycle threshold $(\mathrm{Ct})$ value from SRT-PCR for influenza A was 27.4 (standard deviation $(\mathrm{sd})=5, n=250$ ) and for influenza B was 27.2 ( $\mathrm{sd}=5.4, n=69$ ). Among the SRT-PCR positives but MRT-PCR negatives for influenza $\mathrm{A}$, the $\mathrm{Ct}$ values ranged from 21.7 to 39.3 , with most (8 of 10 where data were available) being above 32.4 (which was $1 \mathrm{sd}$ above the influenza A mean), suggesting that these were weak positives. Two of these SRT-PCR influenza A positives but MRT-PCR negatives were also positive for another virus in the multiplex assay (respiratory syncytial virus and human rhinovirus). Among the SRT-PCR positives but MRT-PCR negatives for influenza $\mathrm{B}$, the $\mathrm{Ct}$ values ranged from 22.2 to 38.7 , with most ( 4 of 7 where data were available) being above 32.6 (which was 1 sd above the influenza B mean), suggesting that these were weak positives. Three of these SRTPCR influenza B positives but MRT-PCR negatives were also positive for another virus in the multiplex assay (respiratory syncytial virus and coronavirus). Forty-seven percent of the discordant results and $49 \%$ of the concordant results were from persons who were vaccinated.

The mean Ct value for ribonuclease $\mathrm{P}$ (RNaseP, a protein that may reflect sample quality) for 594 persons who were PCR negative was $28(\mathrm{sd}=2.5)$ versus $29.1(\mathrm{sd}=2.8)$ for those who were PCR positive. The one person who was MRT-PCR positive for influenza A but SRT-PCR negative had a RNaseP value of 24.7, suggesting that the sample quality was good. The RNaseP values for the SRT-PCR positive but MRT-PCR negative ranged from 24.7 to 32.9 , with 3 specimens being higher than 31.9 (a level $>1$ sd above the RNaseP values for the other positives), suggesting that sample quality was good in the majority of such specimens.

\section{Discussion}

For influenza, the multiplex corresponded well with singleplex RT-PCR with high kappa values. In a previous study examining concordance between SRT-PCR and MRT-PCR, Zimmerman et al. [7] reported a kappa of 0.83 (95\% CI $=0.75-0.92$ ) for 2011-2012 when only 3 of the influenza strains identified were influenza B. Some of the discordant samples were positive for another virus in MRT-PCR or weak positives by RT-PCR. The sensitivity ( $96 \%$ for influenza A and 
$91 \%$ for influenza B) and specificity (100\% for influenza A and $100 \%$ for influenza B) were higher in the present study than previously reported (overall sensitivity $=91.1 \%$ and specificity $=98.2 \%$ ) [7]. This might be due to differences in influenza viruses (i.e., a late variation in the predominant H3N2 in 201112 season was noted) [8].

5.1. Strengths and Limitations. This study offers the ability to compare influenza detection using two assays during a winter influenza season in which both $A$ and B strains were circulating. We were unable to test an inclusive sample of all of the ARI specimens with the MRT-PCR. However, the sample size and strength of the sensitivity and specificity analysis were sufficient to allow confidence in the use of the MRT-PCR for detecting both influenza A and B viruses.

\section{Conclusions}

This study supports the similarity between SRT-PCR and MRT-PCR for detecting influenza virus among patients seeking outpatient care for acute respiratory illnesses.

\section{Ethical Approval}

This project was approved by the University of Pittsburgh Institutional Review Board.

\section{Conflict of Interests}

Dr. Zimmerman has research funding from Sanofi Pasteur, Inc., and Pfizer, Inc. Dr. Nowalk has research funding from Pfizer, Inc., and consults for MedImmune, LLC. Mr. Susick has research funding from Sanofi Pasteur, Inc.

\section{Acknowledgments}

The authors would like to thank Alicia Fry, M.D., Jerome I. Tokars, M.D., and Joseph Bresee, M.D., for their editorial assistance. The contents of this paper are solely the responsibility of the authors and do not necessarily represent the official views of the Centers for Disease Control and Prevention or the Department of Health and Human Services. This paper is subject to the CDC's Public Access Policy and should be submitted to PubMed Central. This paper was supported by Cooperative Agreement FOA IP11003, U01IP000467, funded by the Centers for Disease Control and Prevention. The CTSI infrastructure was also supported by the National Institutes of Health through Grant nos. UL1 RR024153 and UL1TR000005.

\section{References}

[1] V. M. Pierce and R. L. Hodinka, "Comparison of the GenMark diagnostics eSensor respiratory viral panel to real-time PCR for detection of respiratory viruses in children," Journal of Clinical Microbiology, vol. 50, no. 11, pp. 3458-3465, 2012.

[2] M. L. Choudhary, S. P. Anand, M. Heydari et al., "Development of a multiplex one step RT-PCR that detects eighteen respiratory viruses in clinical specimens and comparison with real time RTPCR," Journal of Virological Methods, vol. 189, no. 1, pp. 15-19, 2013.

[3] E. W. Orenstein, G. de Serres, M. J. Haber et al., "Methodologic issues regarding the use of three observational study designs to assess influenza vaccine effectiveness," International Journal of Epidemiology, vol. 36, no. 3, pp. 623-631, 2007.

[4] J. J. Treanor, H. K. Talbot, S. E. Ohmit et al., "Effectiveness of seasonal influenza vaccines in the United States during a season with circulation of all three vaccine strains," Clinical Infectious Diseases, vol. 55, no. 7, pp. 951-959, 2012.

[5] Centers for Disease Control and Prevention, "Early estimates of seasonal influenza vaccine effectiveness-United States, January 2013," Morbidity and Mortality Weekly Report, vol. 62, pp. 32-35, 2013.

[6] S. K. Sanghavi, A. Bullotta, S. Husain, and C. R. Rinaldo, "Clinical evaluation of multiplex real-time PCR panels for rapid detection of respiratory viral infections," Journal of Medical Virology, vol. 84, no. 1, pp. 162-169, 2012.

[7] R. K. Zimmerman, C. R. Rinaldo, M. P. Nowalk et al., "Influenza and other respiratory virus infections in outpatients with medically attended acute respiratory infection during the 201112 influenza season," Influenza and Other Respiratory Viruses, vol. 8, no. 4, pp. 397-405, 2014.

[8] S. E. Ohmit, M. G. Thompson, J. G. Petrie et al., "Influenza vaccine effectiveness in the 2011-2012 season: protection against each circulating virus and the effect of prior vaccination on estimates," Clinical Infectious Diseases, vol. 58, no. 3, pp. 319-327, 2014. 

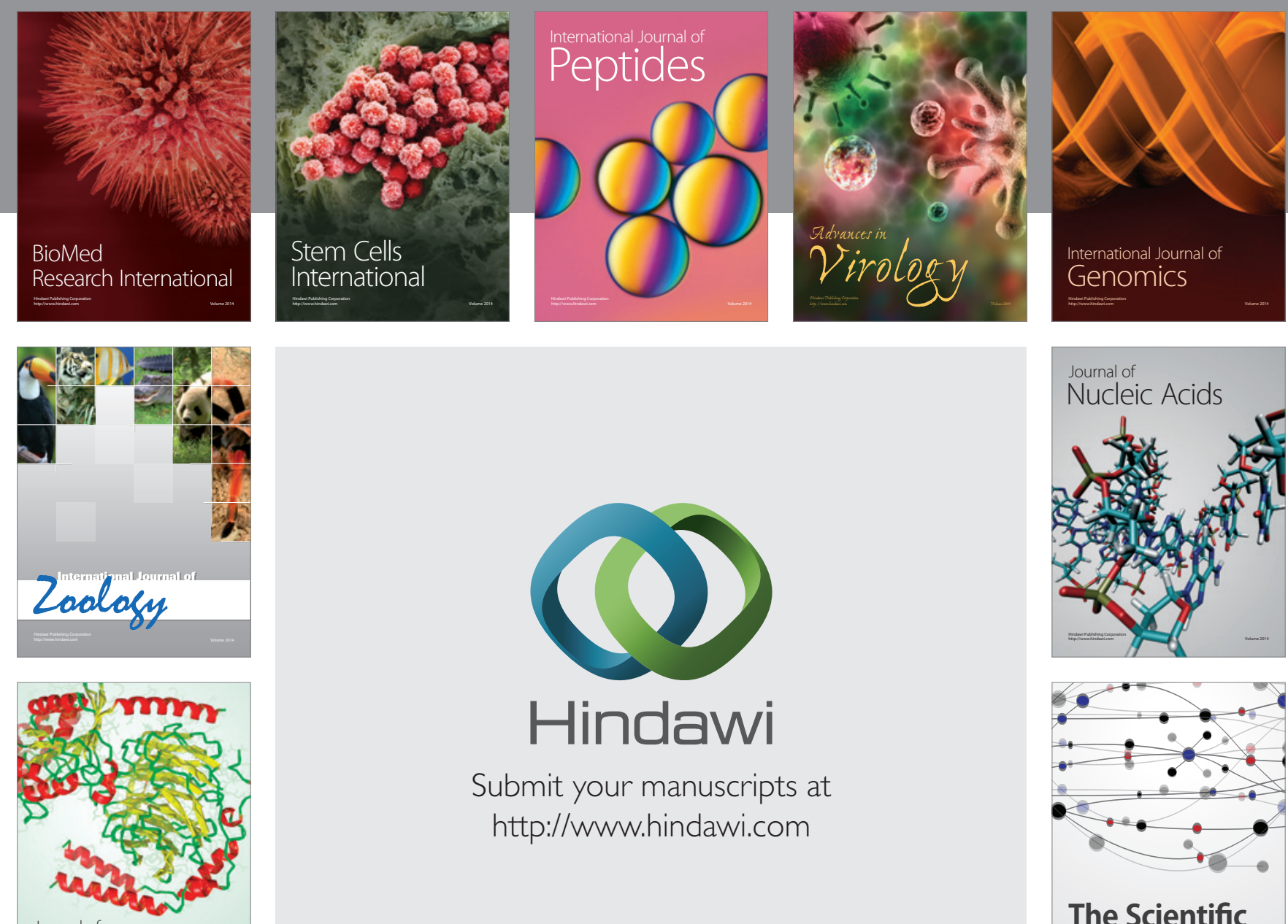

Submit your manuscripts at

http://www.hindawi.com

Journal of
Signal Transduction
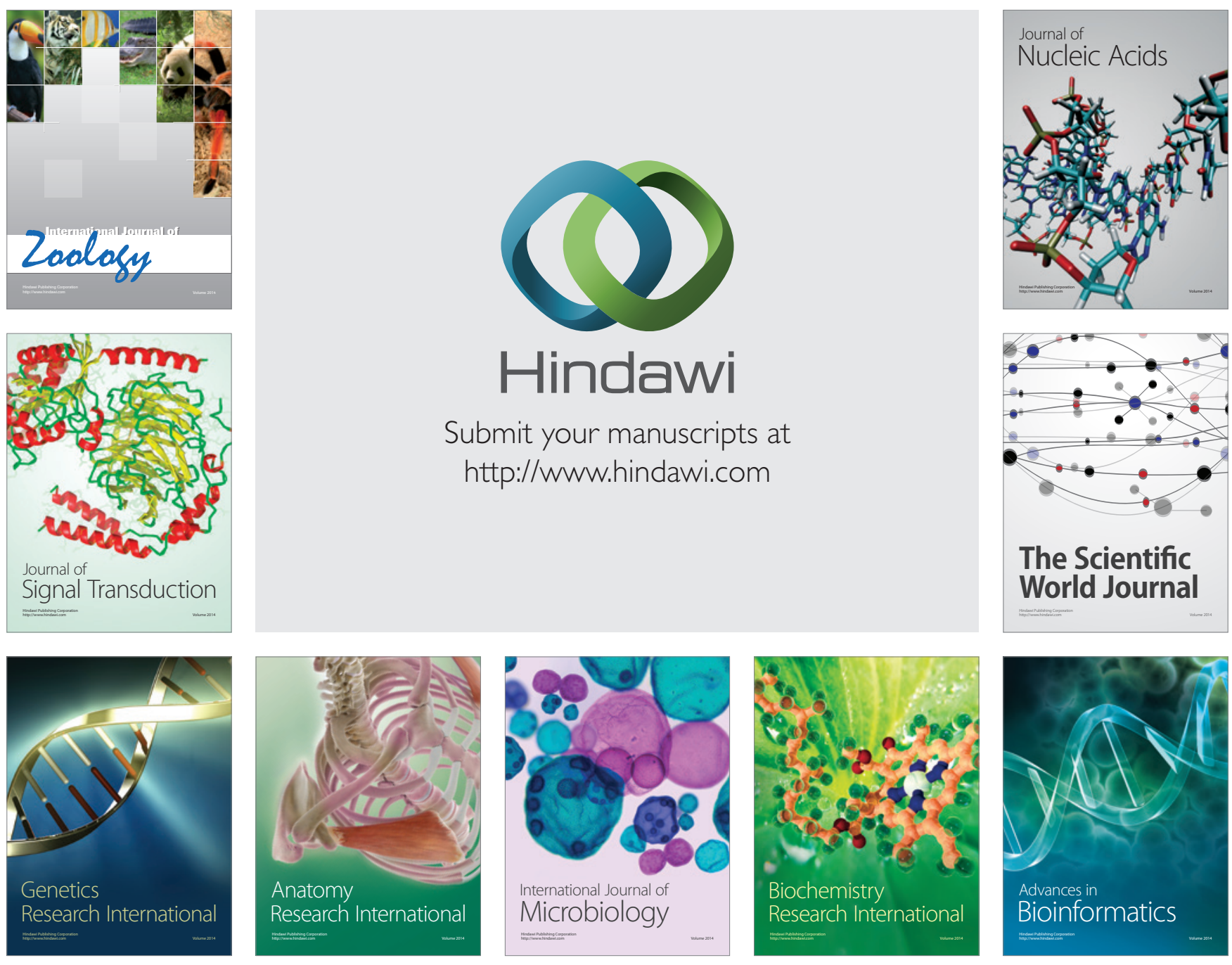

The Scientific World Journal
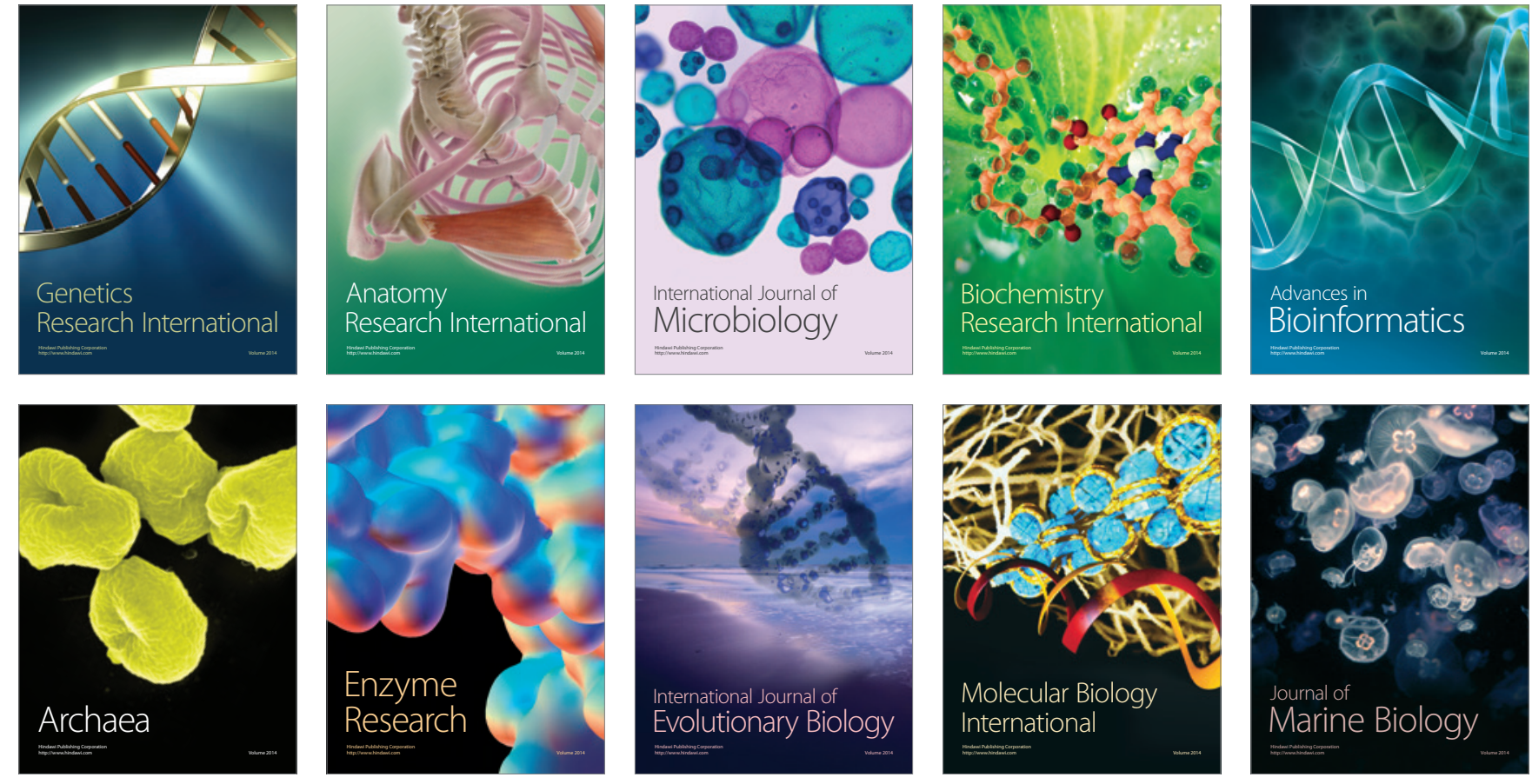